\title{
Perioperative immunomodulation with interleukin-2 in patients with renal cell carcinoma: results of a controlled phase II trial
}

\author{
T Klatte', A Ittenson' ${ }^{2}$, F-W Röhl', M Ecke ${ }^{4}$, EP Allhoff' and M Böhm ${ }^{*, 1}$ \\ 'Department of Urology, Otto-von-Guericke-University, Leipziger Straße 44, 39120 Magdeburg, Germany; ${ }^{2}$ Institute of Immunology, Otto-von-Guericke- \\ University, Leipziger Straße 44, 39120 Magdeburg, Germany; ${ }^{3}$ Institute of Biometrics and Medical Informatics, Otto-von-Guericke-University, Leipziger \\ Straße 44, 39I 20 Magdeburg, Germany; ${ }^{4}$ Department of Urology, Städtisches Klinikum, Birkenallee 34, 39I 30 Magdeburg, Germany
}

\begin{abstract}
We conducted a non-randomised controlled phase II trial to investigate the role of preoperative administration of interleukin-2 (IL-2) in patients with renal cell carcinoma undergoing tumour nephrectomy. A total of 120 consecutive patients were allocated alternately to the two study groups: perioperative immunomodulation with IL-2 (IL-2 group; $n=60)$ and perioperative immunomonitoring without immunomodulation (control group; $n=60$ ). Patients from the $\mathrm{IL}-2$ group received four doses of $10 \times 10^{6} \mathrm{IU} \mathrm{m} \mathrm{m}^{-2}$ twice daily subcutaneously a week before operation followed by a daily maintenance dose of $3 \times 10^{6} \mathrm{IU} \mathrm{m}^{-2}$ subcutaneously until a day before the operation. Parameters of cellular and humoral immunity (leucocytes, T-cell markers CD3, CD4, and CD8, B-cell marker CDI9, monocyte marker CDI4, natural killer (NK) cell markers CD 16, CD56, and CD57, activation markers CD6, CD25, CD28, and CD69, progenitor cell marker CD34, as well as IL-2, IL-6, IL- I 0, soluble IL-2 receptor, IL-I receptor antagonist, transforming growth factor$\beta 1$, and vascular endothelial growth factor) were measured in peripheral venous blood at various intervals. Interleukin-2-related toxicity was WHO grade I (24\%), 2 (67\%), and 3 (9\%). In the postoperative period, T-cell markers, activation markers, and NK cell markers decreased, and IL-6 and IL- 10 increased. However, all these alterations were significantly less accentuated in patients who had been pretreated with IL-2. Median follow-up was 40 months. Tumour-specific survival in the IL-2 group and the control group was 98 vs $81 \%$ after I year and 86 vs $73 \%$ after 5 years $(P=0.04)$. A similar effect was found for progression-free survival. We conclude that IL-2 can be safely administered in the perioperative period and modulates immunological parameters. However, to validate the survival data, a larger randomised phase III trial is needed.
\end{abstract}

British Journal of Cancer (2006) 95, I |67- | 173. doi:I0.1038/sj.bjc.660339| www.bjcancer.com

Published online 10 October 2006

(c) 2006 Cancer Research UK

Keywords: renal cancer; interleukin-2; immunotherapy; surgery; survival

Renal cell carcinoma (RCC) accounts for $3 \%$ of all adult malignancies. Over 38000 new cases and 12000 deaths were estimated for 2006 in the United States (Jemal et al, 2006). Surgery is a mainstay of therapy for both localised and metastasised RCC. However, surgery is associated with considerable perioperative dysfunction of the immune system (Böhm et al, 2001). Also, circulating tumour cells are frequently found in patients with RCC (McKiernan et al, 1999; Ashida et al, 2000; Bilkenroth et al, 2001; Shimazui et al, 2004) and are more prevalent in the renal vein and after surgery (Ashida et al, 2000), possibly owing to surgeryassociated dissemination of tumour cells into the blood circulation, and the presence of circulating tumour cells appears to be associated with a poor prognosis (Shimazui et al, 2004).

Considered an immunosensitive disease, RCC is being treated with various immunotherapeutic regimens both before and after surgery. So far, no survival benefit has been demonstrated (Pizzocaro et al, 2001; Clark et al, 2003; Messing et al, 2003; Atzpodien et al, 2005; Bex et al, 2006). In these studies, no immunotherapy was administered during the perioperative period

*Correspondence: Dr M Böhm; E-mail: malte.boehm@microdissect.de Received 29 June 2006; revised 21 August 2006; accepted 21 August 2006; published online 10 October 2006 when immunodysfunction is most pronounced and circulating tumour cells are most frequently detected.

On this background, we conducted a prospective controlled phase II trial to assess the effect of immunomodulation with interleukin-2 (IL-2) in the potentially critical perioperative period in patients with RCC undergoing tumour nephrectomy.

\section{PATIENTS AND METHODS}

\section{Study objectives and patient eligibility}

Patients with a renal tumour who were scheduled for radical nephrectomy at our institution were eligible for entry into this phase II trial. Each patient met the following eligibility criteria: (a) Eastern Cooperative Oncology Group performance status (ECOG PS) of $\leqslant 2$; (b) life expectancy $>3$ months; (c) age at least 18 years; (d) adequate bone marrow, hepatic, and renal function; and (e) negative pregnancy test for women of childbearing potential. Exclusion criteria were as follows: no histologically proven RCC; prior or synchronous malignancies; autoimmune diseases; pregnancy or lactation; uncontrolled infections; chronic debilitating diseases; positive HIV status; and the presence of contraindications to IL-2. 
Informed consent was obtained from 120 consecutive patients. They were allocated alternately to the two study groups: perioperative immunomodulation with IL-2 (IL-2 group; $n=60$ ) and perioperative immunomonitoring without immunomodulation as a control group (control group; $n=60$ ). The study was approved by the local ethics committee.

\section{Treatment and toxicity assessment}

Recombinant human IL-2 (Aldesleukin, Chiron, Ratingen, Germany) was administered subcutaneously for 6 days 1 week before the operation starting with four initial high-dose injections of $10 \times 10^{6}$ international units (IU) $\mathrm{m}^{-2}$ twice daily followed by a daily maintenance dose of $3 \times 10^{6} \mathrm{IU} \mathrm{m}^{-2}$ until 1 day before the operation. The maintenance but not the initial dose was reduced if toxicity was encountered. Co-medication during the initial high IL-2 dose phase included the antipyretic metamizole (1 g every $4 \mathrm{~h}$ ) and the antimemetic metoclopramide on demand. Interleukin-2related toxicity was determined in a standardised way according to the WHO classification: mild (grade 1), moderate (grade 2), and severe (grade 3 ). Toxicity was monitored until 10 days after the operation.

\section{Immunological analyses}

Peripheral venous blood was collected before and during the administration of IL-2, in both study groups 1 day before and immediately after the operation and on the first, third, fifth, and tenth postoperative day. The blood sample was drawn early in the morning after overnight fasting by puncture of an antecubital vein of the arm.

Parameters in cellular and humoral immunity, including differential blood count, T-cell markers CD3, CD4, and CD8, Bcell marker CD19, monocyte marker CD14, natural killer (NK) cell markers CD16, CD56, and CD57, activation markers CD6, CD25, CD28, CD69, and HLA-DR, progenitor cell marker CD34, and cytokine IL-1 receptor antagonist (IL-1-RA), IL-2, soluble IL-2 receptor (sIL2-R), IL-6, IL-10, transforming growth factor (TGF)$\beta 1$, and vascular endothelial growth factor (VEGF), were measured using standard technique and commercially available kits. Cellular parameters: Whole blood (100 $\mu \mathrm{l}$, EDTA) was stained with monoclonal antibodies conjugated with fluorescein isothiocyanate (Becton Dickinson, Heidelberg, Germany), phycoerythrin (Becton Dickinson), or PC5 (R-phycoerythrincyanin 5.1, Immunotech, Marseille, France). Erythrocytes were lysed by incubation with FACS lysing solution (Becton Dickinson, Heidelberg, Germany) for $15 \mathrm{~min}$, and leucocyte subsets were determined by flow cytometry using a FACS Calibur (Becton Dickinson) with CellQuest Pro software (Becton Dickinson). Cytokines: Citrate-supplemented tubes were used and processed for plasma within $1 \mathrm{~h}$. After centrifugation ( 3000 r.p.m., $10 \mathrm{~min}$ ) and removal of the plasma, the samples were stored at $-80^{\circ} \mathrm{C}$ until analysis. Cytokines were analysed using commercial quantitative enzyme-linked immunoassays (Quantikine Human Immunoassays, R\&D Systems $\mathrm{GmbH}$, Wiesbaden, Germany).

\section{Follow-up and statistical analyses}

Follow-up was surveyed according to the guidelines of the European Association of Urology beginning 4-6 weeks after surgery. Data are presented as the mean \pm standard error of the mean (s.e.m.). Both univariate and multivariate analyses were performed depending on the data subset and question to be answered. Whenever possible, multivariate analyses were performed in order to validate univariate statistics. Characteristics of the two treatment groups were compared using the $\chi^{2}$-test and the Student's $t$-test for categorical and continuous variables, respectively. The primary end points were the alterations in parameters of cellular and humoral immunity. Secondary end points included toxicity, tumour-specific survival, and time to progression. Survival curves were determined using the Kaplan-Meier method and calculated from the date of nephrectomy. For univariate survival analysis, survival curves were compared using the logrank test. It should, however, be emphasised that extensive subanalyses were limited because of the size of the patient population. Nonetheless, we address survival analysis in patients with stage IV RCC separately because of the different treatment modalities these patients received. For multivariate survival analysis, a Cox regression analysis was performed. Statistical analysis was carried out using the SPSS (version 11.5) program. In all analyses, the significance level was specified as $P \leqslant 0.05$.

\section{RESULTS}

Between May 1999 and September 2004, 120 consecutive patients were enrolled. Four patients were withdrawn because histology showed that they had no RCC. Therefore, the intention-to-treat population consisted of 116 patients, with 58 in each study group. The two study groups did not differ with respect to age, sex, ECOG PS, tumour stage, tumour diameter, Thoenes grade, histological subtype, location, operation time, operative technique, or perioperative blood transfusions. Table 1 summarises the patient and tumour characteristics.

\section{Toxicity}

All patients who received IL-2 suffered from IL-2-related toxicity grade $1(24 \%), 2(67 \%)$, or $3(9 \%)$. Toxicity subsided usually the day after the last high-dose IL-2 injection. Relevant toxicity criteria are shown in Table 2 .

\section{Immunological analyses}

For reasons of clarity and brevity, data are presented as plots. Cellular markers and cytokines are shown in Figures 1 and 2, respectively. Mean \pm s.e.m. are indicated.

During IL-2 treatment, T-cell markers (CD3, CD4, CD8), activation markers (CD6, CD25, CD28, HLA-DR), B-cell marker (CD19), and NK cell markers (CD16, CD56, CD57) decreased significantly (day $-7 v s-6$ ), followed by an increase at the day of operation. Progenitor cell marker CD34 did not change from day -7 to day -6 , but was significantly lower in the IL-2 group on day -1 . In the postoperative period, all patients showed elevated leucocyte and granulocyte but decreased lymphocyte counts, T-cell markers, activation markers, and NK cell markers. However, all these alterations were significantly less pronounced in patients treated with IL-2. In contrast to their preoperative levels, the postoperative kinetics of B-cell marker CD19 and progenitor cell marker CD34 was not significantly different between both groups. Monocyte marker CD14 was significantly decreased on day -6 and day 1 in the IL-2 group.

As expected, IL-2 levels were elevated only after administration of IL-2. Interleukin-2 administration was followed by a significant counter-regulation of the soluble IL-2 receptor, IL-1-RA, IL-6, and IL-10. However, the early postoperative increase of IL-6 and IL-10 was significantly less pronounced in treated patients. No changes were found for TGF- $\beta 1$ and VEGF during IL-2 treatment. Both cytokines showed a postoperative decrease, but were not significantly different between the study groups.

\section{Follow-up}

At the time of analysis, 96 patients were alive, 90 of whom had no evidence of disease. The median follow-up for surviving patients was 40 months (range 1-80 months). 
Table I Patient demographic, tumour, and operation characteristics

\begin{tabular}{|c|c|c|c|}
\hline Variable & $\begin{array}{l}\text { IL-2 group } \\
(n=58)\end{array}$ & $\begin{array}{l}\text { Control group } \\
\quad(n=58)\end{array}$ & $P$-value \\
\hline \multicolumn{4}{|l|}{ Age (years) } \\
\hline Median & 64 & 63 & \multirow[t]{2}{*}{0.248} \\
\hline Range & $34-81$ & $36-84$ & \\
\hline \multicolumn{4}{|l|}{ Sex } \\
\hline Female & $20(34 \%)$ & $22(38 \%)$ & \multirow[t]{2}{*}{0.699} \\
\hline Male & $38(66 \%)$ & $36(62 \%)$ & \\
\hline \multicolumn{4}{|l|}{ ECOG PS } \\
\hline 0 & $24(41 \%)$ & $29(50 \%)$ & \multirow[t]{3}{*}{0.530} \\
\hline । & $28(48 \%)$ & $22(38 \%)$ & \\
\hline 2 & $6(10 \%)$ & $7(12 \%)$ & \\
\hline \multicolumn{4}{|c|}{ Tumour stage (2002) } \\
\hline I & $23(40 \%)$ & $24(41 \%)$ & \multirow[t]{4}{*}{0.234} \\
\hline$\|$ & $6(10 \%)$ & $4(7 \%)$ & \\
\hline III & $13(22 \%)$ & $21(36 \%)$ & \\
\hline IV & $16(28 \%)$ & $9(16 \%)$ & \\
\hline \multicolumn{4}{|c|}{ Tumour diameter (mm) } \\
\hline Median & 65 & 55 & \multirow[t]{2}{*}{0.451} \\
\hline Range & $18-200$ & $17-150$ & \\
\hline \multicolumn{4}{|l|}{ Thoenes grade } \\
\hline GI & $9(16 \%)$ & $12(21 \%)$ & \multirow[t]{3}{*}{0.407} \\
\hline $\mathrm{G} 2$ & $37(64 \%)$ & $39(67 \%)$ & \\
\hline G3 & $12(21 \%)$ & $7(12 \%)$ & \\
\hline \multicolumn{4}{|l|}{ Histological subtype } \\
\hline Clear cell & $51(88 \%)$ & $53(9 \mid \%)$ & \multirow[t]{3}{*}{0.504} \\
\hline Papillary & $6(10 \%)$ & $3(5 \%)$ & \\
\hline Chromophobe & I (2\%) & $2(3 \%)$ & \\
\hline \multicolumn{4}{|l|}{ Tumour location } \\
\hline Right-sided & $28(48 \%)$ & $25(43 \%)$ & \multirow[t]{2}{*}{0.313} \\
\hline Left-sided & $30(52 \%)$ & $33(57 \%)$ & \\
\hline \multicolumn{4}{|l|}{ Operation time (min) } \\
\hline Median & 160 & 165 & \multirow[t]{2}{*}{0.864} \\
\hline Range & $70-345$ & $60-380$ & \\
\hline \multicolumn{4}{|l|}{ Type of incision } \\
\hline Transperitoneal & $45(78 \%)$ & $42(72 \%)$ & \multirow[t]{2}{*}{0.520} \\
\hline Flank & $13(22 \%)$ & $16(28 \%)$ & \\
\hline \multicolumn{4}{|c|}{ Blood transfusion, units packed red blood cells } \\
\hline Median & 0 & 0 & \multirow[t]{2}{*}{0.516} \\
\hline Range & $0-6$ & $0-6$ & \\
\hline
\end{tabular}

IL-2 = interleukin-2; ECOG PS = Eastern Cooperative Oncology Group performance status.

For all patients (i.e. patients of all stages), the estimated 1- and 5 -year rates $( \pm$ s.e.m.) of tumour-specific survival were $98 \%$ $( \pm 2 \%)$ and $86 \%( \pm 7 \%)$ in the IL-2 group and $81 \%( \pm 5 \%)$ and $73 \%( \pm 6 \%)$ in the control group, respectively $(P=0.043$; Figure $3 \mathrm{~A}$ ). The median survival time was not reached in either study group. A similar effect was found for progression-free survival: 1 - and 5 -year progression-free survival rates $( \pm$ s.e.m.) for patients were $94 \%( \pm 4 \%)$ and $81 \%( \pm 8 \%)$ in the IL-2 group, and $74 \%( \pm 6 \%)$ and $62 \%( \pm 7 \%)$ in the control group, respectively $(P=0.019$; Figure $3 \mathrm{~B})$. Median time to tumour progression was not reached in either group.

Patients with stage IV disease were addressed separately, because they received the following therapies after nephrectomy: four patients of each group immunochemotherapy (Hanover regimen: IL-2, IFN- $\alpha, 5-\mathrm{FU}$ ), four patients (IL-2 group: three; control group: one) mistletoe treatment, two patients (one of each
Table 2 Interleukin-2-related toxicity according to $\mathrm{WHO}$

\begin{tabular}{lccccc}
\hline & Grade & Grade I & Grade 2 & Grade 3 & Grade 4 \\
\hline Haematological & 58 & 0 & 0 & 0 & 0 \\
Gastrointestinal & & & & & \\
Bilirubin & 58 & 0 & 0 & 0 & 0 \\
Transaminases & 27 & 14 & 12 & 5 & 0 \\
Oral & 53 & 4 & 1 & 0 & 0 \\
Nausea/vomiting & 41 & 9 & 8 & 0 & 0 \\
Diarrhoea & 55 & 3 & 0 & 0 & 0 \\
Constipation & 53 & 5 & 0 & 0 & 0 \\
Renal & & & & & \\
Blood creatinin & 43 & 13 & 2 & 0 & 0 \\
Pulmonary & 58 & 0 & 0 & 0 & 0 \\
Fever with drug & 9 & 15 & 34 & 0 & 0 \\
Allergic & 58 & 0 & 0 & 0 & 0 \\
Cutaneous & 9 & 31 & 18 & 0 & 0 \\
Hair & 58 & 0 & 0 & 0 & 0 \\
Infection & 57 & 1 & 0 & 0 & 0 \\
Cardiac & & & & & \\
Hypotension & 52 & 0 & 6 & 0 & 0 \\
Neurotoxicity & 58 & 0 & 0 & 0 & 0 \\
\hline
\end{tabular}

group) surgical resection of lung metastases, one patient (IL-2 group) IFN- $\alpha$ and vinblastine, and one (IL-2 group) local immunotherapy with inhaled IL-2. The median tumour-specific survival time was $18( \pm 4)$ months in the IL-2 group and $10( \pm 1)$ months in the control group $(P=0.015)$. Median time to progression was $14( \pm 4)$ months and 6 months $( \pm 1)$, respectively $(P=0.006)$. Because of the small patient population (IL-2 group: $n=16$; control group: $n=9$ ), further subanalysis including a separate multivariate analysis was not meaningful.

Notably, we found no difference between the different histological subtypes with respect to perioperative immunodysfunction and survival. A more detailed statistical analysis of subgroups was hampered by the small strata size.

Multivariate analyses were performed using a Cox proportional hazard model. The following covariates were examined: age, ECOG PS, tumour stage, Thoenes grade, histological subtype, treatment with perioperative immunomodulation (yes vs no), leucocytes, CD3, CD4, CD6, CD8, CD16, CD25, CD28, CD56, CD57, CD69, IL1-RA, IL-2, sIL2-R, IL-6, IL-10, TGF- $\beta 1$, and VEGF at different time points. The only variables that reached statistical significance were ECOG PS, tumour stage, Thoenes grade, and perioperative immunomodulation. These parameters are shown in Table 3.

\section{DISCUSSION}

IL-2 is an established and potent immunomodulating agent that exerts a striking array of pleiotropic effects on numerous target cells, the most prominent of which is on T lymphocytes (Gaffen and Liu, 2004). We showed that preoperative treatment with IL-2 effectively modulates perioperative immunodysfunction but causes only moderate short-term toxicity. The early postoperative nadir of cellular parameters of the immune system is less pronounced in IL-2-treated patients. A more detailed discussion of possible cellular mechanisms of this effect can be found elsewhere (Böhm et al, 2002).

Interleukin (IL)-2 modulates not only intravascular but also intratumoral immunity. Recently, Donskov et al (2002, 2004) found a positive correlation between numbers of intratumoral lymphocyte subsets (CD3, CD8, CD57) and both objective response and survival during IL-2-based immunotherapy. It remains to be 

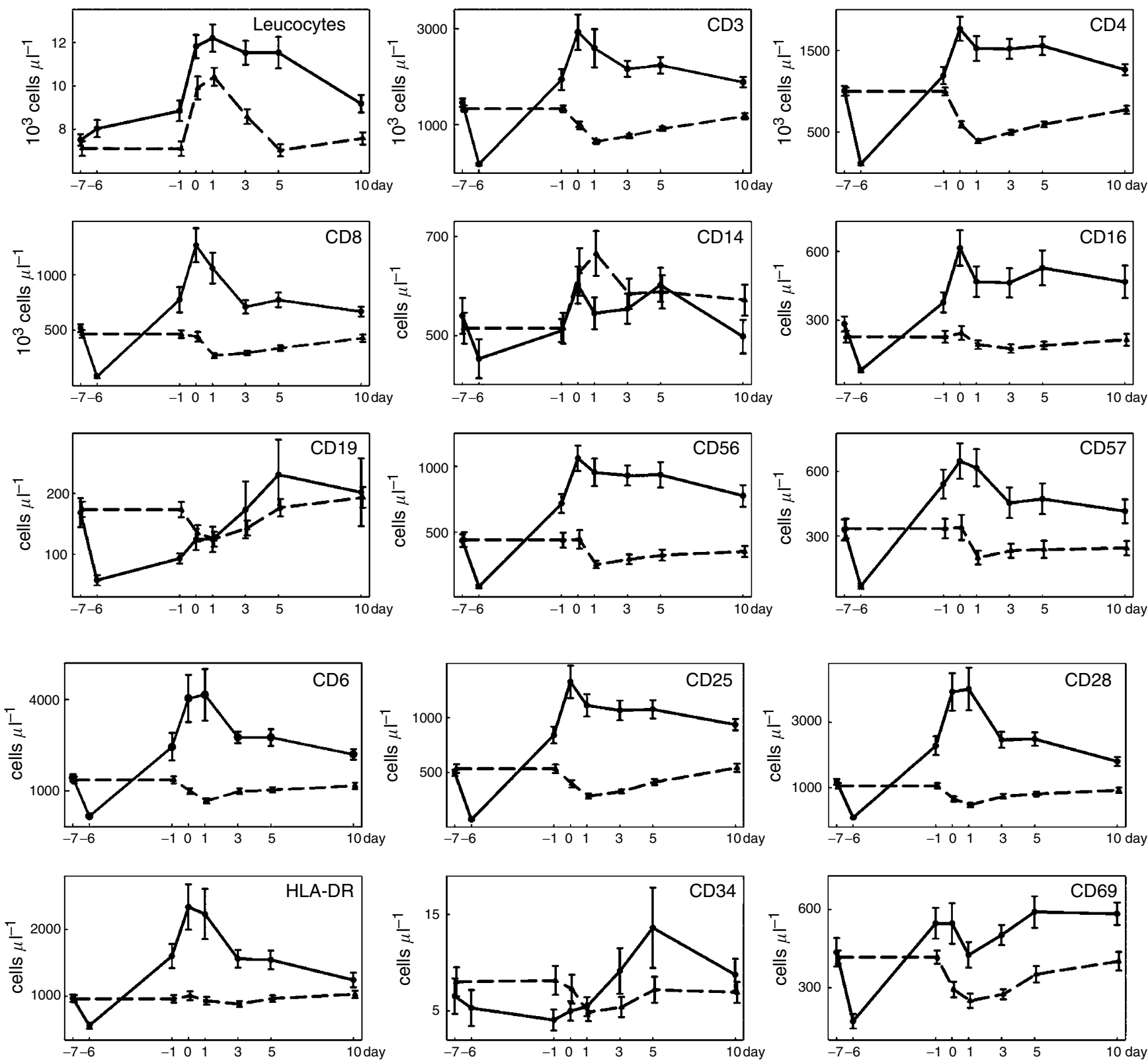

Figure I Perioperative changes of cellular markers. Mean and s.e. are indicated. (- - ): IL-2 group; (-----): control group.

elucidated whether intravascular rather than intratumoral immune function is critical for disease recurrence after nephrectomy.

Administration of IL-2 was started a week before surgery in order to allow the patient's immune system enough time to respond. It was discontinued 1 day before the operation so that IL-2-related toxicity did not interfere with the operation. In many immunotherapeutic regimens, IL-2 is administered over a period of several weeks. During this long period, IL-2-related toxicity is likely to accumulate, which contributes to low patient compliance. In contrast, the much shorter preoperative IL-2 regimen used here was better tolerated. Frequency and severity of side effects were within the reported range for the dosage used (Brivio et al, 1992, 1996). The newly introduced dose decrement after the initial four high doses of IL-2 attenuated a counter-regulation encountered in an earlier regimen (Böhm et al, 2002). Consistent administration of metamizole and metoclopramide increased tolerability. Possible accumulation of IL-2-related toxicity was avoided by limiting the duration of high-dose IL-2 administration to 2 days and overall
IL-2 administration to 1 week. The dose decrement also enhanced the effect of IL-2 pretreatment on the elevation of T-cell, NK cell, and activation markers, and thus on leucocytes reported earlier (Böhm et al, 2002).

With this trial design and the timing of preoperative IL-2 administration, maximum perioperative immunomodulation was achieved in the early postoperative phase when postoperative immunodysfunction is most pronounced. The authors therefore speculate that perioperative as opposed to adjuvant immunotherapy is effective because it modulates the immune system during the most critical perioperative phase when its ability to deal with metastasising tumour cells is at a nadir.

Before the initiation of the study, some thought was spent on its design. Perioperative immunomodulation is an investigational approach and not yet a standard therapeutic option. Therefore, a phase II rather than a phase III trial was designed. A randomised phase III trial with a power of 0.80 and an alpha error of 0.05 would require more than 250 patients in each group. At the present 

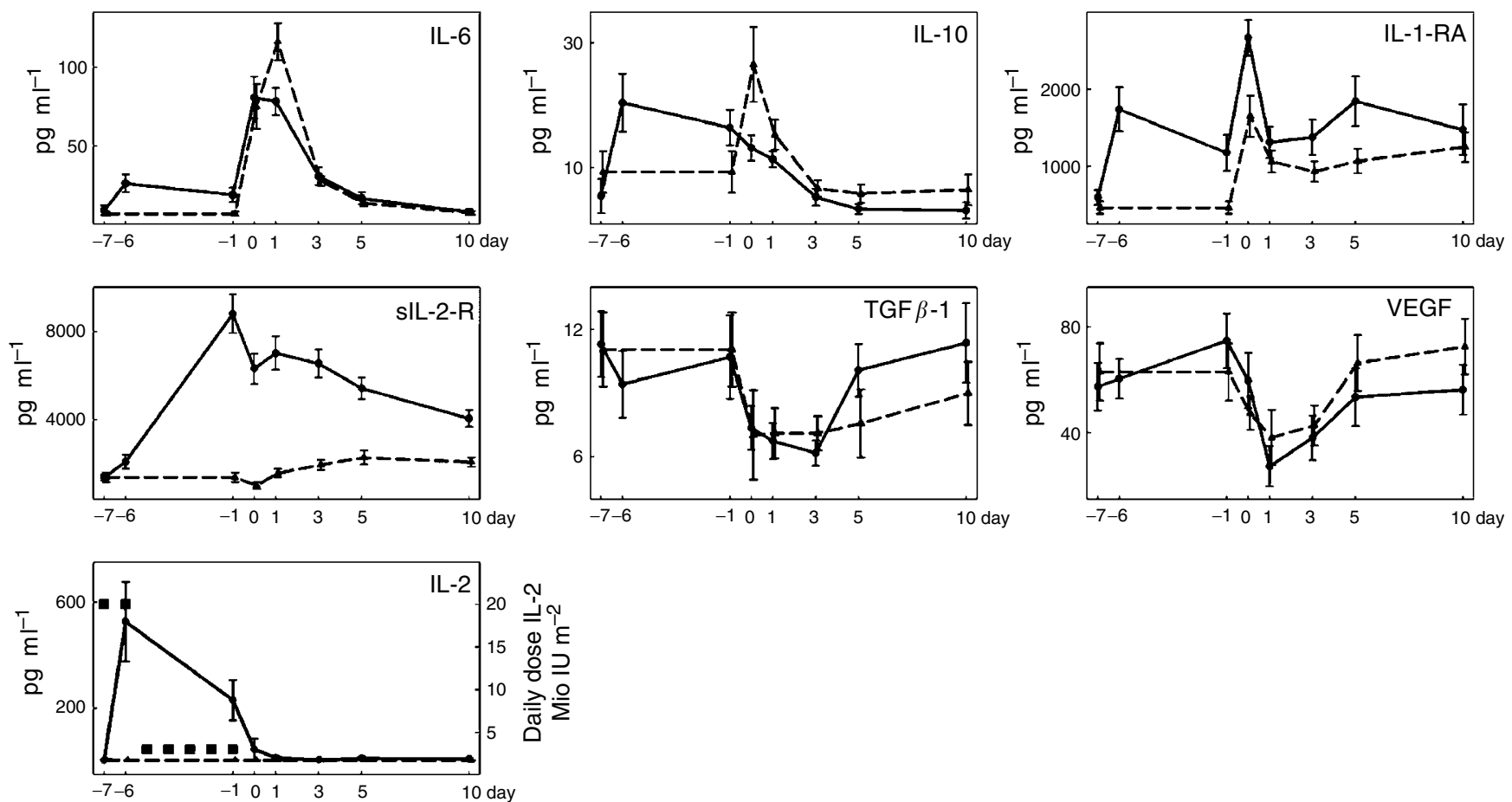

Figure 2 Perioperative changes of cytokines. Presentation of data as in Figure I.
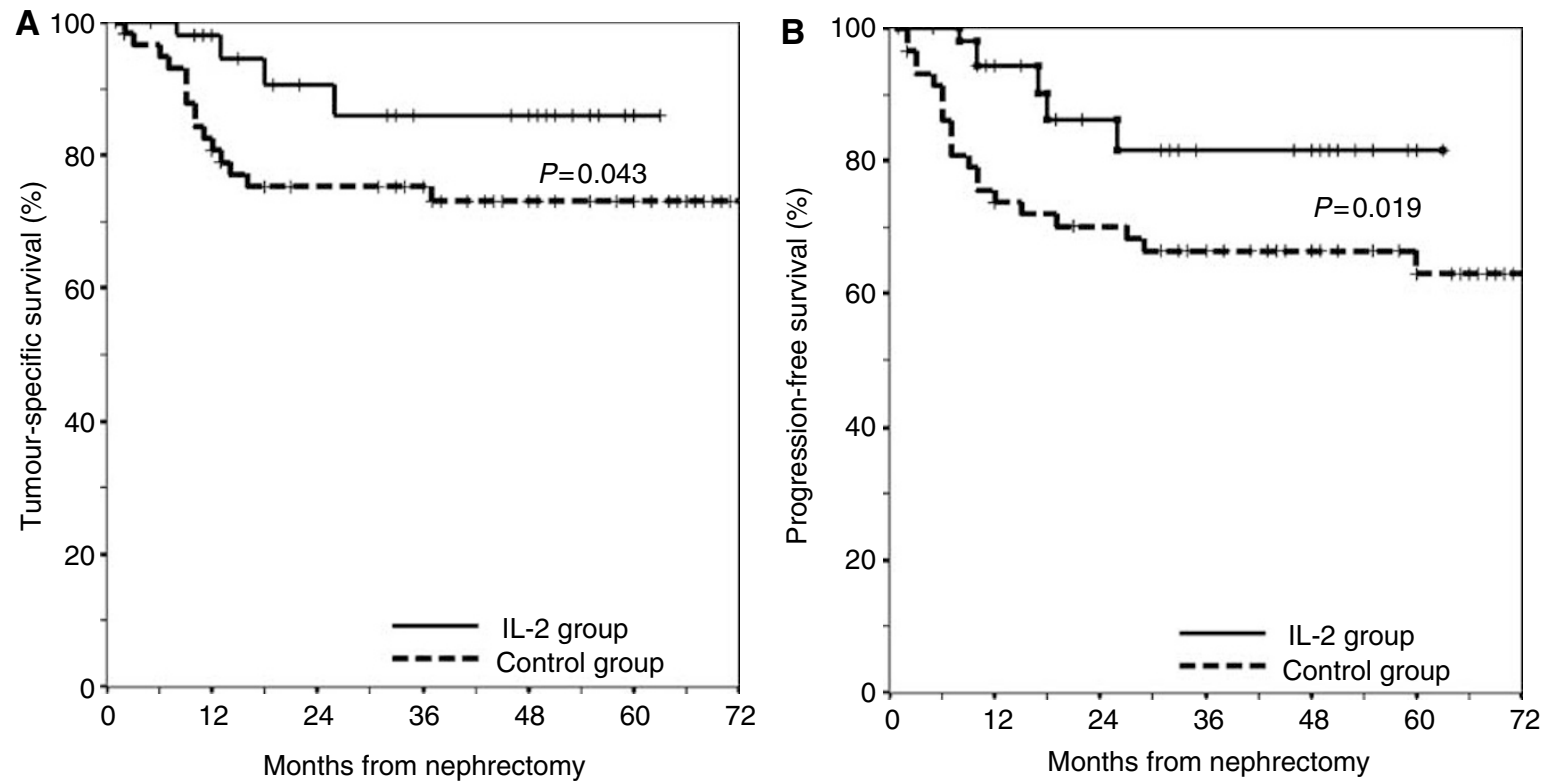

Figure 3 Kaplan-Meier survival estimates of patients treated with IL-2 tumour-specific survival and (B) progression-free survival.

, IL-2 group) and without treatment (----, control group) according to (A)

stage, it appears that it is hardly possible to recruit so many patients, because ethical concerns may arise besides the organisational challenge. To our knowledge, no randomised trial of perioperative immunomodulation has been conducted so far on any malignancy, and the size of the verum group rarely exceeded 20 patients in published studies (Böhm et al, 2003). For these reasons, we opted for a well-controlled non-randomised phase II trial rather than a randomised trial. Keeping also in mind that newer therapeutic approaches focus not only on the immune system but also on other biological pathways such as the VEGF pathway and tyrosine kinase inhibition, a rather broad array of markers was assessed.

It appears that after preoperative administration of IL-2, the perioperative alterations of immunological parameters are most pronounced in the early post-operative period (Böhm et al, 2001). After the 10th post-operative day, these alterations are rather minor (Deehan et al, 1995) and can be biased by other factors. For these reasons, immunological parameters were measured during the first 10 days after surgery. 
Table 3 Cox proportional hazard model

\begin{tabular}{lcc}
\hline Covariate & Hazard ratio (95\% Cl) & P-value \\
\hline ECOG PS & $0.099[0.020-0.495]$ & 0.005 \\
Tumour stage & $0.043[0.007-0.254]$ & 0.001 \\
Thoenes grade & $0.317[0.120-0.837]$ & 0.020 \\
Immunomodulation (yes vs no) & $0.165[0.059-0.463]$ & 0.001 \\
\hline
\end{tabular}

$\mathrm{Cl}=$ confidence interval; ECOG PS=Eastern Cooperative Oncology Group performance status.

Tumour-specific and progression-free survival were longer in IL-2-treated patients. However, this study was not randomised and thus we should not over-interpret the results. We calculated the power of the trial to be 0.17 . To reach a power of 0.80 and an alpha error of 0.05 , we estimated that more than 250 patients would be required in each group. Therefore, we propose that to validate our survival data, future studies incorporate the approach of perioperative immunomodulation whenever possible into prospective, randomised phase III trials.

Two large studies showed that tumour nephrectomy followed by immunotherapy results in longer survival of patients with metastatic renal cell cancer (Flanigan et al, 2001; Mickisch et al, 2001). In our study, no adjuvant immunotherapy was administered, and only five of 16 patients with metastasised (stage IV) RCC received systemic immunotherapy according to departmental

\section{REFERENCES}

Ashida S, Okuda H, Chikazawa M, Tanimura M, Sugita O, Yamamoto Y, Nakamura S, Moriyama M, Shuin T (2000) Detection of circulating cancer cells with von hippel-lindau gene mutation in peripheral blood of patients with renal cell carcinoma. Clin Cancer Res 6: 3817-3822

Atkins MB, Regan M, McDermott D (2004) Update on the role of interleukin 2 and other cytokines in the treatment of patients with stage IV renal carcinoma. Clin Cancer Res 10: 6342S-6346S

Atzpodien J, Royston P, Wandert T, Reitz M (2003) Metastatic renal carcinoma comprehensive prognostic system. Br J Cancer 88: 348-353

Atzpodien J, Schmitt E, Gertenbach U, Fornara P, Heynemann H, Maskow A, Ecke M, Woltjen HH, Jentsch H, Wieland W, Wandert T, Reitz M (2005) Adjuvant treatment with interleukin-2- and interferon-alpha2abased chemoimmunotherapy in renal cell carcinoma post tumour nephrectomy: results of a prospectively randomised trial of the German Cooperative Renal Carcinoma Chemoimmunotherapy Group (DGCIN). Br J Cancer 92: 843-846

Bex A, Kerst M, Mallo H, Meinhardt W, Horenblas S, de Gast GC (2006) Interferon alpha $2 \mathrm{~b}$ as medical selection for nephrectomy in patients with synchronous metastatic renal cell carcinoma: a consecutive study. Eur Urol 49: 76-81

Bilkenroth U, Taubert H, Riemann D, Rebmann U, Heynemann H, Meye A (2001) Detection and enrichment of disseminated renal carcinoma cells from peripheral blood by immunomagnetic cell separation. Int J Cancer 92: $577-582$

Böhm M, Ittenson A, Philipp C, Röhl FW, Ansorge S, Allhoff EP (2001) Complex perioperative immuno-dysfunction in patients with renal cell carcinoma. J Urol 166: $831-836$

Böhm M, Ittenson A, Schierbaum KF, Röhl F-W, Ansorge S, Allhoff EP (2002) Pretreatment with interleukin-2 modulates peri-operative immuno-dysfunction in patients with renal cell carcinoma. Eur Urol 41: $458-468$

Böhm M, Klatte T, Allhoff EP (2003) Perioperative immune modulation in renal cell carcinoma - review of the current situation. Aktuel Urol 34: 319-327

Brivio F, Lissoni P, Alderi G, Barni S, Lavorato F, Fumagalli L (1996) Preoperative interleukin-2 subcutaneous immunotherapy may prolong the survival time in advanced colorectal cancer patients. Oncology 53: 263-268

Brivio F, Lissoni P, Tisi E, Erba L, Barni S, Tancini G, Rescaldani R, Alderi G, Nociti V (1992) Effects of a preoperative therapy with interleukin-2 on guidelines at the time of the trial. This does not warrant subgroup stratification. It also suggests that the survival data reported here are not corrupted by subsequent systemic immunotherapy. Tumour-specific survival in the control group is comparable with that recently reported by other groups (Motzer et al, 1999; Flanigan et al, 2001; Mickisch et al, 2001; Atzpodien et al, 2003; Verra et al, 2003; Atkins et al, 2004), suggesting that a representative and reliable patient population was assessed here.

None of the stage I patients died of recurrent disease, but two of $24(8 \%)$ control patients and none of the IL-2-treated patients had a tumour recurrence during follow-up. Because recurrence is uncommon but does occur among stage I patients (Ljungberg et al, 1999; Gofrit et al, 2001; Pantuck et al, 2001; Zisman et al, 2002), we included these patients in our trial. Owing to the small number of events and the short follow-up, it is not feasible to assess these patients statistically.

In the early postoperative period, TGF- $\beta 1$ and VEGF decreased, but no effect of IL-2 treatment was demonstrated. It appears that IL-2 may not interfere with newer VEGF pathway-targeted tyrosine kinase inhibitor therapies. Possibly, a combination with these therapies may be promising.

Taken together, our results indicate that preoperative treatment with IL-2 may be a feasible, yet investigational approach to complement surgical therapy and modulate some alterations of the immune system that occur perioperatively in patients undergoing tumour nephrectomy. As mentioned above, a larger randomised trial will be needed to validate the survival data. surgery-induced lymphocytopenia in cancer patients. Oncology 49: 215 218

Clark JI, Atkins MB, Urba WJ, Creech S, Figlin RA, Dutcher JP, Flaherty L, Sosman JA, Logan TF, White R, Weiss GR, Redman BG, Tretter CP, McDermott D, Smith JW, Gordon MS, Margolin KA (2003) Adjuvant high-dose bolus interleukin-2 for patients with high-risk renal cell carcinoma: a cytokine working group randomized trial. J Clin Oncol 21: $3133-3140$

Deehan DJ, Heys SD, Ashby J, Eremin O (1995) Interleukin-2 (IL-2) augments host cellular immune reactivity in the perioperative period in patients with malignant disease. Eur J Surg Oncol 21: $16-22$

Donskov F, Bennedsgaard KM, Hokland M, Marcussen N, Fisker R, Madsen HHT, Fode K, von der Maase H (2004) Leukocyte orchestration in blood and tumour tissue following interleukin-2 based immunotherapy in metastatic renal cell carcinoma. Cancer Immunol Immunother 53: 729 739

Donskov F, Bennedsgaard KM, von der Maase H, Marcussen N, Fisker R, Jensen JJ, Naredi P, Hokland M (2002) Intratumoural and peripheral blood lymphocyte subsets in patients with metastatic renal cell carcinoma undergoing interleukin-2 based immunotherapy: association to objective response and survival. $\mathrm{Br} J$ Cancer 87: $194-201$

Flanigan RC, Salmon SE, Blumenstein BA, Bearman SI, Roy V, McGrath PC, Caton Jr JR, Munshi N, Crawford ED (2001) Nephrectomy followed by interferon alfa- $2 \mathrm{~b}$ compared with interferon $\mathrm{alfa}-2 \mathrm{~b}$ alone for metastatic renal-cell cancer. $N$ Engl J Med 345: 1655-1659

Gaffen SL, Liu KD (2004) Overview of interleukin-2 function, production and clinical applications. Cytokine 28: 109-123

Gofrit ON, Shapiro A, Kovalski N, Landau EH, Shenfeld OZ, Pode D (2001) Renal cell carcinoma: evaluation of the 1997 TNM system and recommendations for follow-up after surgery. Eur Urol 39: 669-674

Jemal A, Siegel R, Ward E, Murray T, Xu J, Smigal C, Thun MJ (2006) Cancer statistics, 2006. CA Cancer J Clin 56: 106-130

Ljungberg B, Alamdari FI, Rasmuson T, Roos G (1999) Follow-up guidelines for nonmetastatic renal cell carcinoma based on the occurrence of metastases after radical nephrectomy. Br J Urol Int 84: $405-411$

McKiernan JM, Buttyan R, Bander NH, de la Taille A, Stifelman MD, Emanuel ER, Bagiella E, Rubin MA, Katz AE, Olsson CA, Sawczuk IS 
(1999) The detection of renal carcinoma cells in the peripheral blood with an enhanced reverse transcriptase-polymerase chain reaction assay for MN/CA9. Cancer 86: $492-497$

Messing EM, Manola J, Wilding G, Propert K, Fleischmann J, Crawford ED, Pontes JE, Hahn R, Trump D (2003) Phase III study of interferon alfa-NL as adjuvant treatment for resectable renal cell carcinoma: an Eastern Cooperative Oncology Group/Intergroup trial. J Clin Oncol 21: 12141222

Mickisch GH, Garin A, van Poppel H, de Prijck L, Sylvester R (2001) Radical nephrectomy plus interferon-alfa-based immunotherapy compared with interferon alfa alone in metastatic renal-cell carcinoma: a randomised trial. Lancet 358: 966-970

Motzer RJ, Mazumdar M, Bacik J, Berg W, Amsterdam A, Ferrara J (1999) Survival and prognostic stratification of 670 patients with advanced renal cell carcinoma. J Clin Oncol 17: 2530-2540

Pantuck AJ, Zisman A, Belldegrun AS (2001) The changing natural history of renal cell carcinoma. J Urol 166: 1611-1623
Pizzocaro G, Piva L, Colavita M, Ferri S, Artusi R, Boracchi P, Parmiani G, Marubini E (2001) Interferon adjuvant to radical nephrectomy in Robson stages II and III renal cell carcinoma: a multicentric randomized study. J Clin Oncol 19: 425-431

Shimazui T, Yoshikawa K, Uemura H, Hirao Y, Saga S, Akaza H (2004) The level of cadherin- 6 mRNA in peripheral blood is associated with the site of metastasis and with the subsequent occurrence of metastases in renal cell carcinoma. Cancer 101: $963-968$

Verra N, Jansen R, Groenewegen G, Mallo H, Kersten MJ, Bex A, VythDreese FA, Sein J, van de Kasteele W, Nooijen WJ, de Waal M, Horenblas S, de Gast GC (2003) Immunotherapy with concurrent subcutaneous GM-CSF, low-dose IL- 2 and IFN- $\alpha$ in patients with progressive metastatic renal cell carcinoma. Br J Cancer 88: 1346-1351

Zisman A, Pantuck AJ, Wieder J, Chao DH, Dorey F, Said JW, DeKernion JB, Figlin RA, Belldegrun AS (2002) Risk group assessment and clinical outcome algorithm to predict the natural history of patients with surgically resected renal cell carcinoma. J Clin Oncol 20: 4559-4566 